\title{
Discharge of pursuit neurons in the caudal part of the frontal eye fields during cross-axis vestibular-pursuit training in monkeys
}

\author{
Keishi Fujiwara $\cdot$ Teppei Akao $\cdot$ Sergei Kurkin $\cdot$ \\ Kikuro Fukushima
}

Received: 9 December 2008 / Accepted: 12 March 2009 / Published online: 1 April 2009

(C) The Author(s) 2009. This article is published with open access at Springerlink.com

\begin{abstract}
Previous studies in monkeys have shown that pursuit training during orthogonal whole body rotation results in task-dependent, predictive pursuit eye movements. We examined whether pursuit neurons in the frontal eye fields (FEF) are involved in predictive pursuit induced by vestibular-pursuit training. Two monkeys were rotated horizontally at $20 \%$ for $0.5 \mathrm{~s}$ either rightward or leftward with random inter-trial intervals. This chair motion trajectory was synchronized with orthogonal target motion at $20 \%$ for $0.5 \mathrm{~s}$ either upward or downward. Monkeys were rewarded for pursuing the target. Vertical pursuit eye velocities and discharge of 23 vertical pursuit neurons to vertical target motion were compared before training and during the last $5 \mathrm{~min}$ of the $25-45 \mathrm{~min}$ training. The latencies of discharge modulation of $61 \%$ of the neurons (14/23) shortened after vestibular-pursuit training in association with a shortening of pursuit latency. However, their discharge modulation occurred after $100 \mathrm{~ms}$ following the onset of pursuit eye velocity. Only four neurons $(4 / 23=17 \%)$ discharged before the eye movement onset. A significant change was not observed in eye velocity and FEF pursuit neuron discharge during pursuit alone after training without vestibular stimulation. Vestibular stimulation alone without a target after training induced no clear response. These results suggest that the adaptive change in response to pursuit predic-
\end{abstract}

K. Fujiwara $\cdot$ T. Akao $\cdot$ S. Kurkin $\cdot$ K. Fukushima $(\bowtie)$

Department of Physiology,

Hokkaido University School of Medicine,

West 7, North 15, Sapporo 060-8638, Japan

e-mail: kikuro@med.hokudai.ac.jp

K. Fujiwara

Department of Otolaryngology-Head and Neck Surgery, Hokkaido University School of Medicine,

Sapporo 060-8638, Japan tion was induced by vestibular inputs in the presence of target pursuit. FEF pursuit neurons are unlikely to be involved in the initial stage of generating predictive eye movements. We suggest that they may participate in the maintenance of predictive pursuit.

Keywords Smooth pursuit - Vestibular system . Adaptive change $\cdot$ Prediction $\cdot$ Frontal eye fields . Latency $\cdot$ Pursuit neuron

\section{Introduction}

Moving objects can be accurately tracked by smooth pursuit eye movements that are made in response to motion of the object's image on the retina. Because of the long latencies $(\sim 100 \mathrm{~ms})$ between changes in target movement and the initiation of changes in pursuit eye movements, prediction must compensate for these delays, which are due to processing the visual motion information and/or generating the eye velocity commands during pursuit (e.g., Barnes and Asselman 1991). During movement of the head and/or whole body, the smooth pursuit system does not work independently but interacts with the vestibular system to maintain the precision of eye movements in space (Leigh and Zee 2006 for a review).

Previous studies in our laboratory have indicated that pursuit training with orthogonal whole body rotation in monkeys results in adaptive changes in smooth eye movements. The latencies of smooth eye movements to target motion shorten and initial eye velocities increase in a task-dependent and adaptive manner (Fukushima et al. 2001a, b). Further studies have indicated that the initial adaptive smooth eye movements contain a predictive pursuit component induced by orthogonal vestibular stimulation (Tsubuku et al. 2006). 
The neural substrate for the predictive pursuit induced by cross-axis vestibular-pursuit training is unknown. Potential brain areas are the supplementary eye fields (SEF) and the caudal part of the frontal eye fields (FEF), because these areas contain neurons that discharge during predictive pursuit (SEF: Heinen and Liu 1997; FEF: MacAvoy et al. 1991; Fukushima et al. 2002, 2008) and because both areas receive vestibular inputs (SEF: Fukushima et al. 2004; FEF: Fukushima et al. 2000a; Ebata et al. 2004; Akao et al. 2007, 2009). In particular, the caudal FEF contains smooth pursuit neurons that are thought to issue a pursuit command (for reviews, see Leigh and Zee 2006; Fukushima et al. 2006; also Kurkin et al. 2009).

In the present study, we tested whether pursuit neurons in the caudal FEF discharge during predictive pursuit induced by cross-axis vestibular-pursuit training. We demonstrate that $61 \%$ of FEF pursuit neurons tested exhibited discharge modulation correlated with the change in pursuit eye velocity. However, contrary to our expectation, the great majority of neurons tested $(83 \%)$ discharged after the onset of vertical eye velocity responses during vestibularpursuit training. Some of the results have been presented in preliminary form (Fujiwara et al. 2007).

\section{Materials and methods}

Two monkeys (Sh, Si, Macaca fuscata, 3.5 and $4.5 \mathrm{~kg}$ ) were used. All procedures were performed in strict compliance with the guidelines for the Care and Use of Animals of National Institutes of Health. Specific protocols were approved by the Animal Care and Use Committee of Hokkaido University School of Medicine. Methods for animal preparation, training, and recording were basically similar to those in previous studies (Fukushima et al. 2000a, 2001a; Tsubuku et al. 2006), except for target presentation during chair rotation, and are summarized here briefly.

Each monkey was sedated with ketamine hydrochloride $(5 \mathrm{mg} / \mathrm{kg}$ i.m.), and then anesthetized with pentobarbital sodium $(25 \mathrm{mg} / \mathrm{kg}$ i.p.). Under aseptic conditions, head holders were installed to restrain the head during recording. A scleral search coil was implanted on each eye to record the vertical and horizontal components of eye movement (Fuchs and Robinson 1966). A recording chamber was installed over a craniotomy aiming at Ant. 23 and Lat. 15 stereotaxic coordinates to enable single neuron recording in the peri-arcuate sulcus region as described previously (MacAvoy et al. 1991; Gottlieb et al. 1994; Tanaka and Fukushima 1998; Fukushima et al. 2000a, 2002; Akao et al. 2007, 2009).

The monkeys were trained with apple juice reward for tracking a target spot $\left(0.5^{\circ}\right.$ in diameter $)$ that moved sinusoidally on a 22 -inch computer display (Sony) $(120 \mathrm{~Hz}$ rate) $60 \mathrm{~cm}$ in front of the animals' eyes in an otherwise dark enclosure. Monkeys' heads were firmly restrained in the primate chair in the stereotaxic plane. The monkey chair was fixed to the turntable for horizontal rotation under computer control. The inter-aural midpoint of the animals' head was brought close to the axis of rotation.

\section{Recording procedures}

Extracellular recordings were made in the left peri-arcuate sulcus region to search for neurons related to pursuit of a moving target spot as reported previously (Tanaka and Fukushima 1998; Fukushima et al. 2000a, 2002, 2008; Akao et al. 2007, 2009). Once an isolated neuron that responded during pursuit was encountered, smooth pursuit responses were tested in eight directions (vertical, horizontal and 2 oblique planes at $45^{\circ}$ angles) to determine the preferred direction. Once single neurons responding to vertical smooth pursuit were isolated, the monkeys were subjected to cross-axis vestibular-pursuit training.

Figure 1a illustrates schematically the stimulus conditions. Monkeys were rotated horizontally at $20 \%$ s for 0.5 s either rightward or leftward with a trapezoidal (i.e., velocity ramp) trajectory [Fig. 1a, chair pos $(\mathrm{H})$, chair vel $(\mathrm{H})]$. The chair remained stationary during the random-duration inter-trial interval (1-3 s, arrows, Fig. 1a). This chair motion trajectory was synchronized with orthogonal (i.e., vertical) target motion at $20 \%$ for $0.5 \mathrm{~s}$ either upward or downward [Fig. 1a, target pos (V)]. Rightward chair rotation was usually combined with upward target motion (Fig. 1a, the first chair movement). To synchronize horizontal chair rotation with vertical target motion, the vertical target position was controlled using a position signal derived from the chair motion that was obtained as a potentiometer output of the horizontal turntable. In addition, the target moved horizontally at $5 \%$ either toward right or left for $\pm 10^{\circ}$ during training [Fig. 1a, target pos $(\mathrm{H})]$. As a result, identical horizontal chair rotation (e.g., toward right) was combined with upward/rightward or upward/leftward target motion (Fig. 1a). All position signals were recorded on a data recorder (Teac, DAT data recorder RD145T), and when target motion was applied alone without vestibular stimulation, we used these signals by replaying the tape in order to give the identical target position signals (i.e., vertical target motion combined with a slow horizontal component). In previous cross-axis vestibular-pursuit training (Fukushima et al. 2001a, b; Tsubuku et al. 2006), slow horizontal target motion [Fig. 1a, target pos $(\mathrm{H})$ ] was not added. We added this component in the present study to exclude any possible interference from fixation (Goldreich et al. 1992; Schwartz and Lisberger 1994; Fukushima et al. 1996a; Izawa et al. 2004).

Daily training lasted for $45 \mathrm{~min}$ in 15 min blocks in each training session 4 days a week. Each 45 min training session evoked a total of 750 chair rotation either toward right 

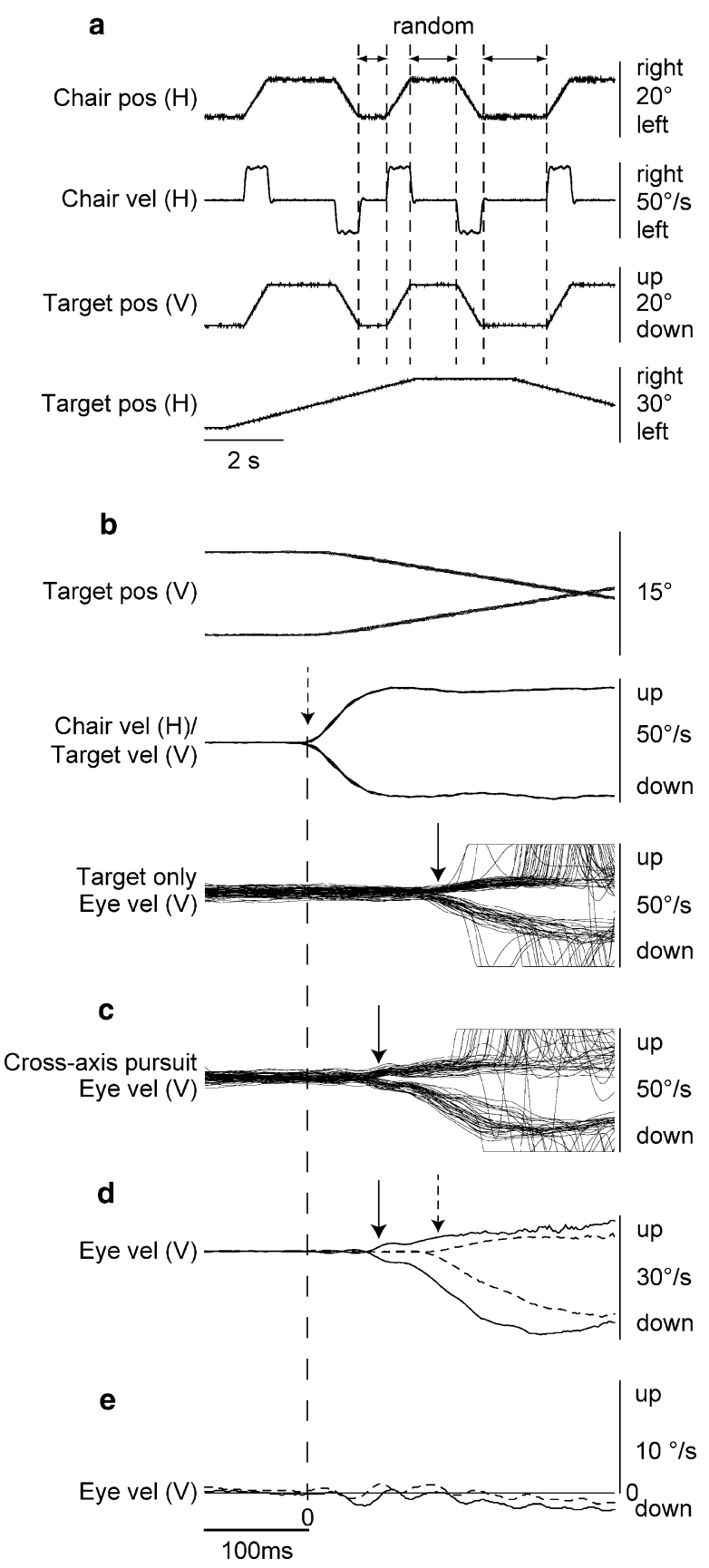

or left (Fig. 1a). In previous studies, direction of chair rotation (either toward right or left) and target motion direction (either toward up or down) were fixed (e.g., right/up) and they counted how many of identical combinations were repeated during training (Tsubuku et al. 2006). In the present study, however, identical horizontal chair rotation (e.g., toward right) was combined with upward/rightward or upward/leftward target motion due to addition of the slow horizontal component (Fig. 1a). We, therefore, counted number of trials as the total number of horizontal chair rotation in the present study (e.g., Fig. 2c, d). The total number
Fig. 1 Stimulus trajectory and representative vertical pursuit eye movements before and after training for cross-axis vestibular-pursuit. a Trajectory of stimuli for horizontal chair position and velocity [chair pos $(\mathrm{H})$, chair vel $(\mathrm{H})]$ and vertical target position [target pos $(\mathrm{V})]$ and horizontal target position [target pos $(\mathrm{H})]$. b Vertical target position and velocity, and/or horizontal chair velocity, and vertical eye velocity [Eye vel (V)] induced by target motion alone before training. $\mathbf{c}$ Vertical eye velocity induced by vertical target motion synchronized with horizontal chair rotation during the last $5 \mathrm{~min}$ of training. In $\mathbf{b}$ and $\mathbf{c}$, saccade velocities were clipped, and all traces (upward and downward target and eye velocity) were superimposed and aligned on the onset of target motion indicated by dashed arrow in b. d Compares de-saccaded mean vertical eye velocity to target motion alone before training (dashed) and during the last 5 min of training with vestibular stimulation (solid). Arrows indicate the onset of vertical eye velocity during the two conditions. e Compares de-saccaded mean vertical eye velocity during chair rotation alone before (dashed) and after training (sol$i d$ ) in complete darkness without a target. In e, vertical eye velocity during the initial $100 \mathrm{~ms}$ before the onset of target motion was subtracted. In $\mathbf{c}-\mathbf{e}$, the onset of chair rotation is indicated by a dashed line. For further explanation, see text

of trials in the present study is two times larger than the number of trials in Tsubuku et al. (2006).

Training effects were examined by comparing responses before training without chair rotation to the last $5 \mathrm{~min}$ of the training period with chair rotation. Before training, identical target motion (vertical motion combined with a slow horizontal component, Fig. 1a) was used without chair rotation. We examined both ocular tracking performance and the discharge of individual neurons to the vertical component of target motion (i.e., vertical target motion). Response to vestibular stimulation was examined by comparing eye velocity and discharge of individual neurons during chair rotation without a target in complete darkness before and after training. Training effects were also examined after training by comparing ocular tracking performance and discharge of individual neurons induced by identical target motion without chair rotation.

\section{Data analysis}

Eye-, target-, and chair-position signals were digitized at $500 \mathrm{~Hz}$ using a 16-bit A/D board (National Instruments) on a Macintosh Quadra computer. These position signals were differentiated by analog circuits (DC-100 Hz, $-12 \mathrm{~dB} /$ octave) to obtain velocity. Eye velocity in the present study indicates eye-in-head velocity. Neural discharge was discriminated, detected at $100 \mathrm{kHz}$, and stored in temporal register with analog signals. Saccades were marked with a cursor on eye velocity traces and removed using an interactive computer program as described previously (Singh et al. 1981; Fukushima et al. 2000a). To examine the latency of neuronal discharge to vertical target motion, we first aligned 20-40 trials on the stimulus 
Fig. 2 Time course of latency changes in vertical eye velocity responses and discharge modulation of FEF pursuit neurons induced by vestibular-pursuit training. a De-saccaded, mean upward and downward eye velocity. b Discharge modulation (raster and histogram) of an upward pursuit neuron. All traces were aligned on the onset of vertical target motion. Arrows indicate latencies of eye velocity (a) and discharge modulation (b). Time course of latency changes of eye velocity responses and simultaneously recorded FEF pursuit neurons are summarized in $\mathbf{c}$ and $\mathbf{d}$, respectively. Thick lines in $\mathbf{c}$ and $\mathbf{d}$ pot data shown in $\mathbf{a}$ and $\mathbf{b}$. Thin and dashed lines in $\mathbf{c}$ and $\mathbf{d}$ pot latencies of two other neurons (d) and simultaneously recorded vertical eye velocity (c). In a-d, $0 \mathrm{~ms}$ indicates the time at which vertical target motion was applied with (0-5 to 30-35 min) and without (before) horizontal chair rotation a
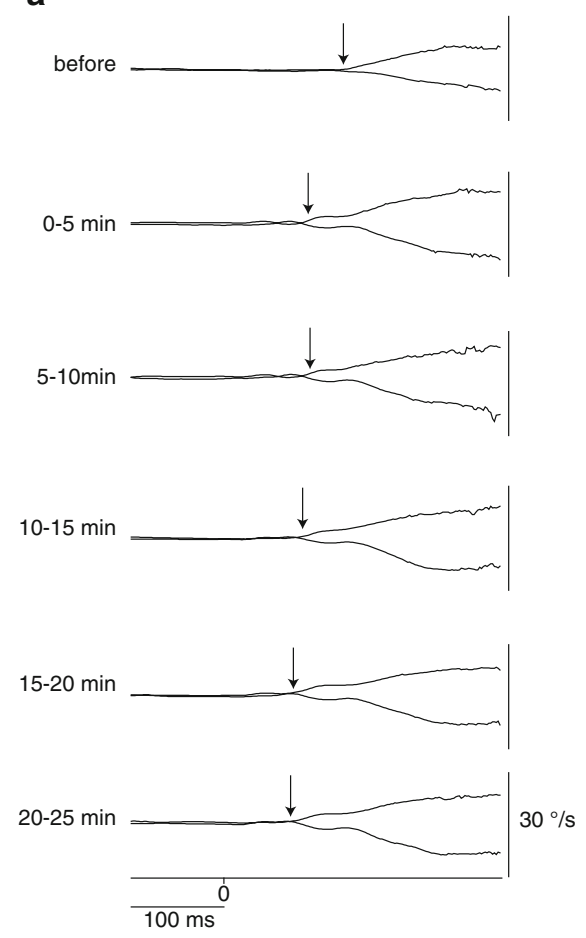

C

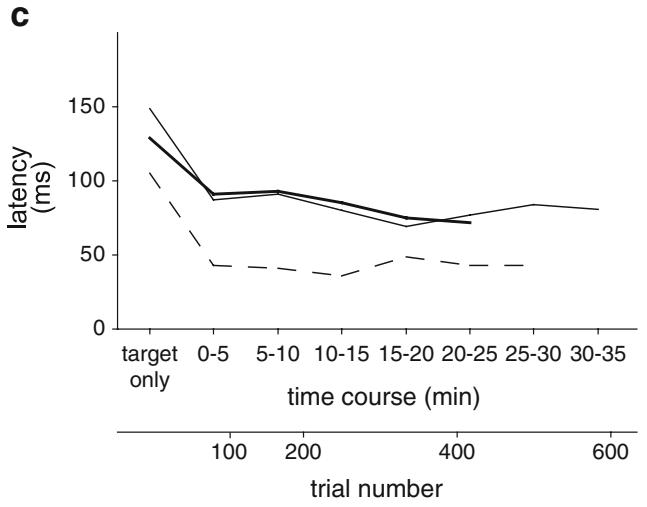

b

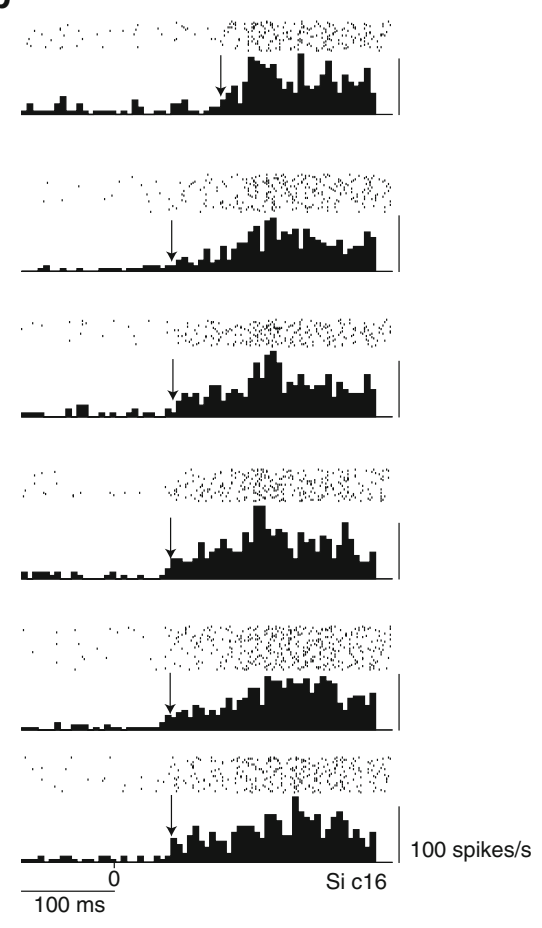

d

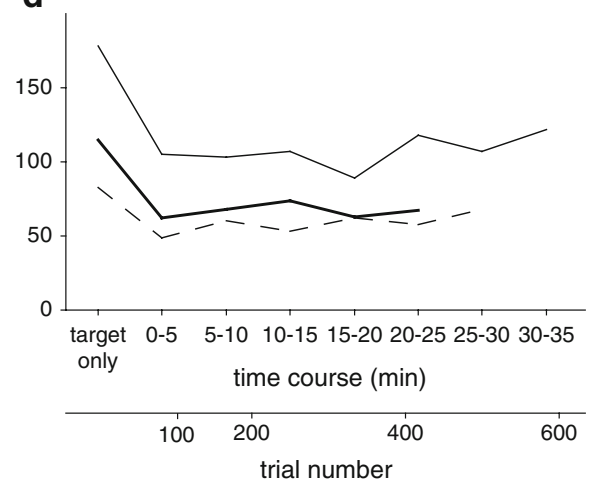

(i.e., vertical target and/or chair motion) onset. Because discharge may have been affected by saccades, we then omitted all traces in which saccades appeared within $\sim 100 \mathrm{~ms}$ of the stimulus onset. A spike density function (SDF) was constructed from spike trains of individual neurons as the sum of Gaussian functions (unit area, $17.6 \mathrm{~ms}$ sigma) centered on the individual spike events (Szucs 1998; Richmond et al. 1987; Richmond and Optican 1987). Pre-stimulus baseline values (mean and standard deviations, SD) for SDF were calculated from the $100 \mathrm{~ms}$ interval immediately prior to stimulus onset. This mean value was subtracted from the discharge modulation. Onset of the neuronal response to the stimulus motion was defined as the time at which the mean discharge rate exceeded $2 \mathrm{SD}$ of the control value (e.g., Akao et al. 2005a). Latencies of eye velocity responses were measured as the time at which the mean for oppositely directed eye velocities diverged (e.g., Fukushima et al. 2001a). Latencies of neuronal discharge to the onset of vertical eye movements were also examined by aligning 20-40 trials on the onset of vertical eye velocity responses with or without chair rotation. Students' $t$ test was used to examine statistical significance $(p<0.05)$.

\section{Histological procedures}

Near the conclusion of recordings, the recording sites of monkey $\mathrm{Si}$ were verified histologically as previously described (e.g., Tanaka and Fukushima 1998; Fukushima et al. 2000a, 2002; Akao et al. 2009). 


\section{Results}

Change in latency and magnitude of pursuit eye movements induced by cross-axis vestibular-pursuit training

Figure $1 \mathrm{~b}, \mathrm{c}$ illustrates representative vertical eye velocity of one monkey (Sh) in response to the vertical component of target motion [target vel $(\mathrm{V})$ ] before training without vestibular stimulation (Fig. 1b) and during the last $5 \mathrm{~min}$ of the 45 min cross-axis vestibular-pursuit training (Fig. 1c). Upward and downward eye velocity traces for upward and downward target motion were superimposed (Fig. 1b, c). Compared to vertical eye velocity before training [Fig. 1b, eye vel $(\mathrm{V})]$, latencies of vertical eye velocity during the last $5 \mathrm{~min}$ of vestibular-pursuit training were clearly shorter (eye vel in Fig. 1c, arrow vs. eye vel in Fig. 1b, arrow). Notice that identical target motion (vertical motion combined with a slow horizontal component, Fig. 1a) was used in Fig. 1b, c except that in Fig. 1c horizontal chair rotation was added (see "Materials and methods"). Mean latencies of vertical eye velocity to the onset of target motion during the two conditions (Fig. 1b-d) were 126 and $72 \mathrm{~ms}$, respectively. Initial vertical eye velocity also increased (Fig. 1d). For comparison, Fig. 1e shows mean de-saccaded vertical eye velocity during horizontal vestibular stimulation alone without a target in complete darkness before and after 45 min training (dashed and solid lines, respectively). Orthogonal vestibular stimulation alone without a target induced no clear vertical eye movement responses before and after training (Fig. 1e, see "Discussion").

Figure 2a illustrates the time course of the change in mean upward and downward eye velocity of another monkey (Si). Latencies of vertical eye velocity responses (Fig. 2a, arrows) to target motion are summarized in Fig. 2c for this recording session (thick line) and two other sessions (thin and dashed lines). Latencies clearly shortened within 20 min of training. Thus, using the present task condition (Fig. 1a), latencies of pursuit eye movements shortened and initial eye velocities increased (Figs. 1d, 2a, c), similar to previous studies (Fukushima et al. 2001a; Tsubuku et al. 2006). These results suggest that similar neural mechanisms were involved in predictive pursuit eye movements induced by cross-axis vestibular-pursuit training in previous and present studies.

Discharge of FEF pursuit neurons during cross-axis vestibular-pursuit raining

Previous studies indicate that preferred directions for individual pursuit neurons in the caudal FEF are distributed nearly evenly for all directions (MacAvoy et al. 1991; Gottlieb et al. 1994; Tanaka and Fukushima 1998; Fukushima et al. 2000a). In the present study, we determined the pre- ferred directions of 105 pursuit neurons in the caudal FEF by testing smooth pursuit responses in eight directions (vertical, horizontal and 2 oblique planes at $45^{\circ}$ angles, see "Materials and methods"). About one-fourth of them (23/ $105=22 \%$ ) had vertical preferred directions. These included 13 upward pursuit neurons and 10 downward pursuit neurons. Cross-axis vestibular-pursuit training was tested for these neurons for $45 \mathrm{~min}$, although some neurons $(n=3)$ were lost after $25 \mathrm{~min}$ of training. Of the 23 neurons, 11 were recorded from monkey Sh and 12 were recorded from monkey Si. Discharge characteristics of the neurons recorded in the two monkeys were similar.

Figure $2 b$ illustrates the time course of the change in initial discharge modulation of a single neuron during training. Simultaneously recorded vertical eye velocities are shown in Fig. 2a (see above). Latencies of discharge modulation of this neuron to target motion are summarized in Fig. 2d (thick line) together with two other neurons (Fig. 2d, thin and dashed lines). Latencies of discharge modulation clearly shortened within $20 \mathrm{~min}$ of training, especially during the initial $5 \mathrm{~min}$ of training (Fig. 2d). It should be mentioned that during training some FEF pursuit neurons exhibited periodicity in discharge rate as illustrated in Fig. 2b (20-25 min). Such a periodicity was observed in 6 of 21 (29\%) neurons checked in both monkeys. The periodicity in discharge modulation had frequencies ranging from 30 to $50 \mathrm{~Hz}$ (peak to peak discharge interval 20-33 ms) (see "Discussion").

To compare the change in initial discharge modulation and eye velocity responses further, Fig. 3 shows discharge of a representative neuron during pursuit before training (a) and during the last $5 \mathrm{~min}$ of training (b). Traces were aligned on the onset of target motion (Fig. 3a, b, $0 \mathrm{~ms}$ ). The onsets of vertical pursuit eye velocity and discharge modulation are indicated by arrows. This neuron discharged with the latency of $83 \mathrm{~ms}$ after the onset of target motion before training (Fig. 3a, arrow). During the last $5 \mathrm{~min}$ of training, it discharged with the latency of $49 \mathrm{~ms}$ (Fig. 3b, arrow). But the latency of vertical eye velocity responses was even shorter (Fig. 3b). For comparison, in Fig. 3c the same traces were aligned with the onset of vertical pursuit eye velocity responses $(0 \mathrm{~ms})$. After training, this neuron discharged $22 \mathrm{~ms}$ after the onset of vertical eye velocity. Horizontal chair rotation alone without a target in complete darkness induced minimal discharge before and after training [Fig. 3d, chair vel (H)]. Notice that chair rotation was applied in the plane orthogonal to the pursuit preferred directions.

Of the 23 FEF pursuit neurons tested, the majority (14/ $23=61 \%$ ) exhibited shorter discharge latencies following vertical target motion during the last $5 \mathrm{~min}$ of training. These included nine up pursuit neurons and five down pursuit neurons. Figure 4a plots the difference in latencies for 


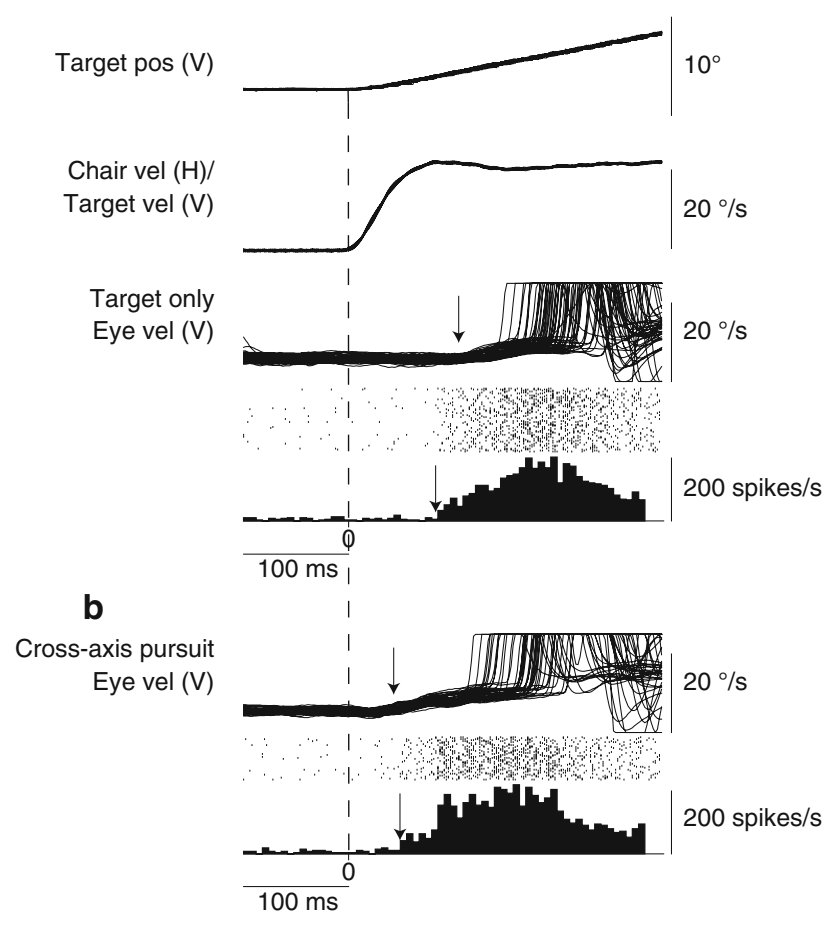

C

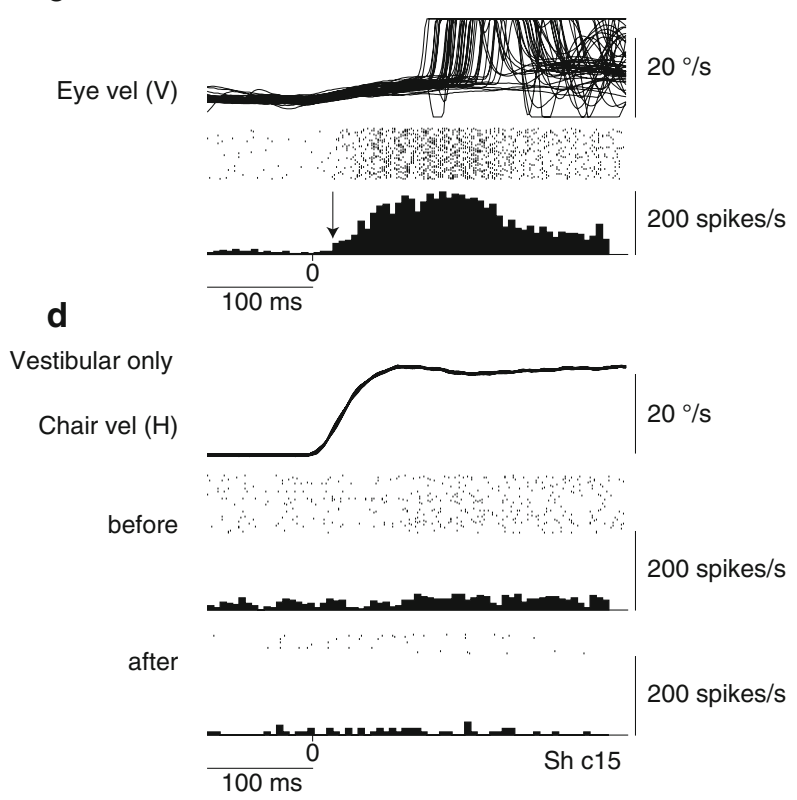

Fig. 3 Discharge of a representative FEF pursuit neuron before and after cross-axis vestibular-pursuit training. a Vertical target position and velocity, and/or horizontal chair velocity, and superimposed vertical eye velocity and neuronal discharge (raster and histogram) induced by target motion alone before training. $\mathbf{b}$ Vertical eye velocity and discharge of the same neuron induced by combination of horizontal rotation and vertical target motion during the last $5 \mathrm{~min}$ of training. c The same trials as $\mathbf{b}$. All traces in $\mathbf{c}$ were aligned on the onset of vertical eye velocity responses. Arrows indicate onsets of eye velocity responses and discharge modulation. In $\mathbf{a}-\mathbf{c}$, saccade velocities are clipped. d Compares discharge of this neuron during chair rotation alone without a target before training and after training the 14 neurons following the onset of target motion before training without vestibular stimulation (before) and during the last $5 \mathrm{~min}$ of the training period with vestibular stimulation (after). On average, latencies shortened by $38 \mathrm{~ms}$ after training (Fig. 4a, circle). Figure 4b plots latency difference for simultaneously recorded vertical eye velocity. Latencies of vertical eye velocity were shorter by a mean of $55 \mathrm{~ms}$ after training (Fig. 4b, before vs. after). The mean latency shortening was larger for eye velocity than for the discharge of FEF pursuit neurons (Fig. 4a vs. b). For comparison, Fig. 4c plots latencies of the 14 neurons relative to the onset of vertical eye velocity responses when discharge modulation was aligned on the onset of vertical eye velocity before and after training (e.g., Fig. 3c). The majority $(10 / 14=71 \%)$ of these neurons discharged after the onset of eye velocity responses after training with the mean latency of $12 \mathrm{~ms}$ lag (Fig. 4c, after).

The remaining nine neurons $(9 / 23=39 \%)$ did not exhibit latency shortening during vestibular-pursuit training. Latencies of these neurons to the onset of target motion are plotted in Fig. 4d together with simultaneously recorded vertical eye velocities (Fig. 4e). Latencies of two of the 9 neurons could not be determined because of large SDs during initial fixation after training, and are not plotted in Fig. 4d. Although the mean vertical eye velocities exhibited similar shortening of latencies to target motion synchronized with horizontal chair rotation after training (Fig. 4e vs. b, circles), latencies of these seven neurons increased with the mean of $29 \mathrm{~ms}$ after training (Fig. 4d, before vs. after). The mean latency of these neurons to the onset of vertical eye velocity responses was $68 \mathrm{~ms}$ lag (Fig. 4f).

To further examine the effects of cross-axis vestibularpursuit training on the discharge of FEF pursuit neurons, we compared the magnitude of discharge modulation during the initial $200 \mathrm{~ms}$ of vertical pursuit after the onset of target motion before training and during the last 5 min of training with horizontal chair rotation. For the discharge during the last 5 min of training, discharge during chair rotation alone in complete darkness was subtracted to minimize vestibular only related discharge modulation. Figure 5a is an example of a neuron that exhibited latency shortening after training. We first calculated mean discharge modulation during the initial $200 \mathrm{~ms}$ after the onset of target motion before and after training (i.e., the blue and red shaded areas in Fig. 5a, respectively). Figure 5c plots mean discharge rates for each of the 14 neurons during this period before training (before) and during the last $5 \mathrm{~min}$ of training (after). These neurons exhibited shorter latencies during the last $5 \mathrm{~min}$ of the training period (Fig. 4a). Nearly all 14 neurons (12/14) increased their mean discharge rate during the initial $200 \mathrm{~ms}$ (Fig. 5c). Overall means for the 14 neurons before and after training were 18 and $25 \mathrm{sp} / \mathrm{s}$, 

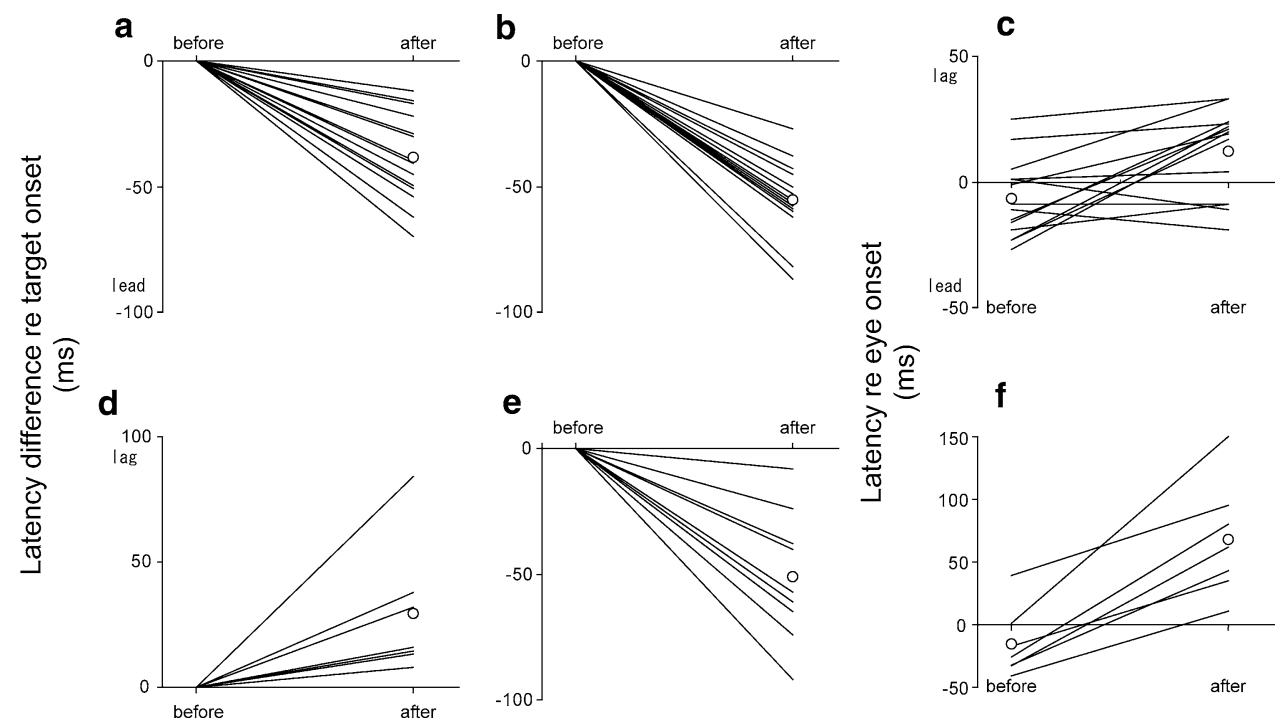

Fig. 4 Change in latency of discharge modulation of FEF pursuit neurons and vertical eye velocity before and after cross-axis vestibularpursuit training. Before indicates latencies before training. After indicates latencies during the last $5 \mathrm{~min}$ of training with horizontal whole body rotation. Latencies of discharge modulation $(\mathbf{a}, \mathbf{d})$ and vertical eye velocity responses $(\mathbf{b}, \mathbf{e})$ after training were calculated relative to the onset of vertical target motion. In $\mathbf{c}$ and $\mathbf{f}$, latencies of discharge modulation were calculated relative to the onset of vertical eye velocity responses before and after training. Difference in latencies of discharge

respectively (Fig. 5c, circles). The difference between before and after training was significant ( $p<0.05$, Fig. $5 c)$.

For comparison, we calculated the magnitude of simultaneously recorded vertical eye velocity over initial $200 \mathrm{~ms}$ as illustrated in Fig. 5b (the blue and red shaded areas), and plotted the mean eye velocity in Fig. $5 \mathrm{~d}$ for each of the 14 neurons. The magnitude of eye velocity also increased after training while recording these responsive neurons. Overall mean eye velocities while recording these 14 neurons before and after training were 0.7 and $2.7 \%$, respectively (Fig. $5 \mathrm{~d}$, circles). The difference between before and after training was significant (Fig. 5d, $p<0.05$ ).

Because after training the majority of these 14 neurons (10/14) discharged after the onset of eye velocity (Fig. 4c), we also compared initial discharge modulation during $100 \mathrm{~ms}$ after the onset of eye velocity responses. The results are plotted in Fig. 5e. In most neurons tested (10/ 14), the initial discharge modulation during $100 \mathrm{~ms}$ after the onset of vertical eye velocity decreased. The difference between before and after training was significant (Fig. 5e, $p<0.05$ ).

We further compared discharge modulation during 100$200 \mathrm{~ms}$ after the onset of vertical eye velocity responses before and after training. These neurons indeed showed significant increase in discharge modulation during this period. However, vertical eye velocity responses during this period did not show a significant change after training, modulation of $14 \mathrm{FEF}$ pursuit neurons that exhibited decreased latencies (a) and simultaneously recorded vertical eye velocity (b). c Plots latencies of discharge modulation of the 14 neurons. d Plots difference in latencies of discharge modulation for seven of nine FEF pursuit neurons that did not exhibit shortening of latencies of discharge modulation. e Difference in latencies of simultaneously recorded vertical smooth eye movements. f Plots latencies of discharge modulation of the seven neurons. Open circles in $\mathbf{a}-\mathbf{f}$ indicate group mean values. For further explanation, see text

because the change was mostly latencies relative to the onset of target motion and not in the eye velocity trajectory (e.g., Fig. 5b, g). These results indicate that discharge of these neurons minimally contributed to the initial $100 \mathrm{~ms}$ of eye velocity responses after training (see "Discussion").

None of the remaining nine neurons that did not exhibit latency shortening during the last $5 \mathrm{~min}$ of the training (Fig. 4d), showed an increase in the mean discharge rate during the initial $200 \mathrm{~ms}$ of target motion. Figure $5 \mathrm{f} \mathrm{com-}$ pares discharge modulation of a representative neuron before and after training (blue and red, respectively). Discharge modulation of seven of the nine neurons even decreased when compared with the discharge before training (Fig. 5h) despite the fact that the magnitude of the mean vertical eye velocity increased after training while recording these neurons (Fig. 5i). Overall mean discharge modulation for the nine neurons before and after training were 8 and $4 \mathrm{sp} / \mathrm{s}$, respectively (Fig. $5 \mathrm{~h}$, circles, $p<0.05$ ). Overall mean eye velocities before and after training while recording these neurons were 0.8 and $2.3 \%$, respectively (Fig. 5i, $p<0.05)$.

Discharge of FEF pursuit neurons during spot motion alone before and after cross-axis vestibular-pursuit training

We examined whether there was a change in discharge modulation during pursuit after training in the absence of 
a

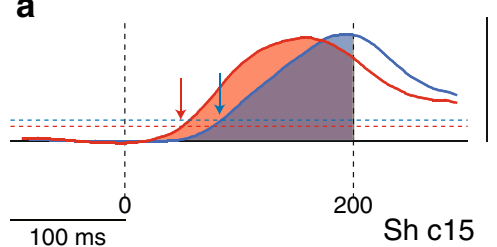

b
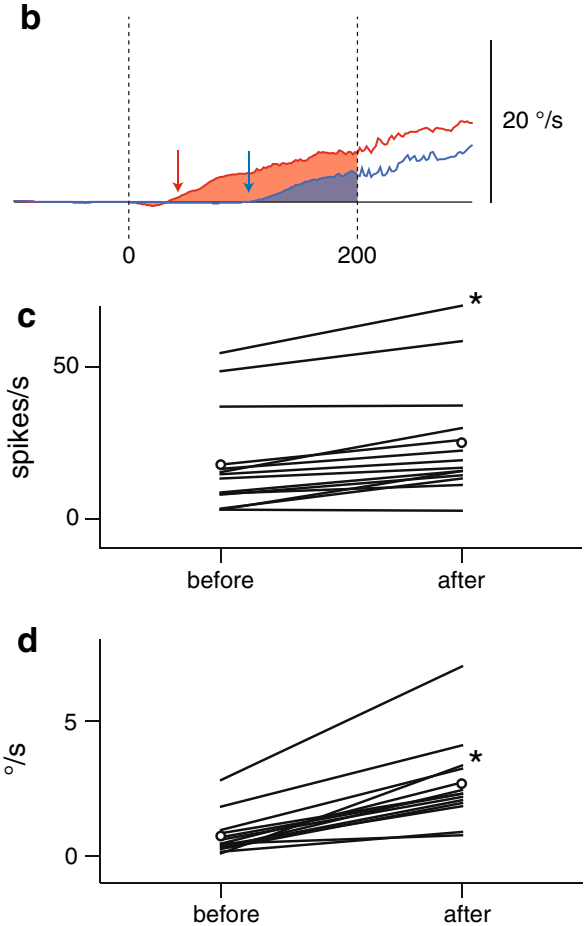

e

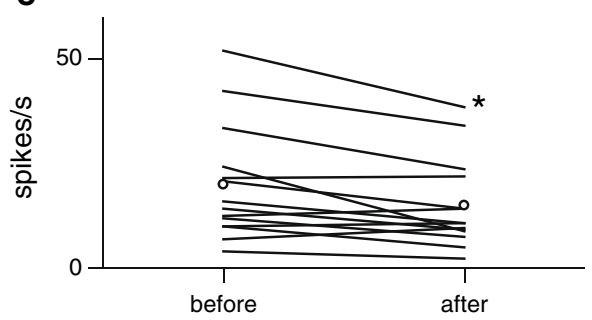

Fig. 5 Comparison of magnitude of discharge modulation in FEF pursuit neurons and simultaneously recorded vertical eye velocity. In a-d and $\mathbf{f}-\mathbf{i}$, spike density function (SDF) and de-saccaded, mean vertical eye velocity were compared during initial $200 \mathrm{~ms}$ after target motion onset before training (before) and during the last $5 \mathrm{~min}$ of training (after). SDF of a representative neuron (a) and simultaneously recorded vertical eye velocity (b), discharge of 14 neurons that exhibited latency shortening after training (c) and simultaneously recorded vertical eye velocity (d). In $\mathbf{a}$ and $\mathbf{b}$, blue and red indicate averaged traces before and after training, respectively. In c, discharge modulation before and after training was significantly different $(p<0.05)$. In $\mathbf{d}$, vertical eye velocity before and after training was significantly different $(p<0.05)$. In $\mathbf{e}, \mathrm{SDF}$ of the same neurons were compared during the initial $100 \mathrm{~ms}$ after the onset of vertical eye velocity before and after training. SDF of another neuron (f), simultaneously recorded vertical eye velocity (g),

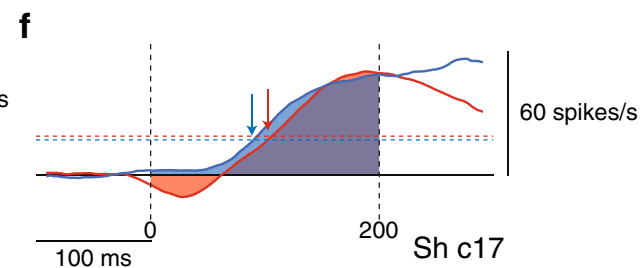

g

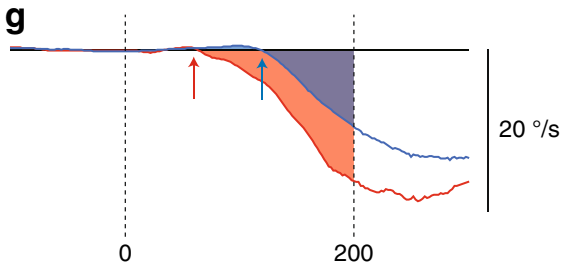

h

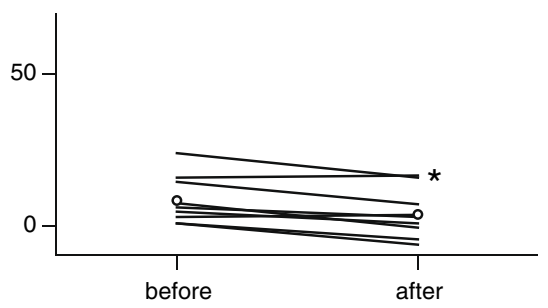

i

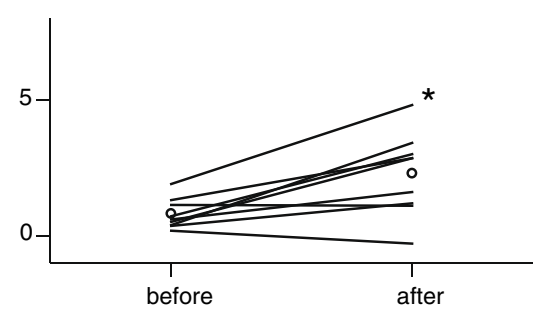

discharge of nine neurons that did not exhibit latency shortening after training (h), and simultaneously recorded vertical eye velocity (i). In $\mathbf{f}$ and $\mathbf{g}$, blue and red indicate averaged traces before and after training, respectively. In $\mathbf{h}$, discharge modulation before and after training was significantly different $(p<0.05)$. In $\mathbf{i}$, vertical eye velocity before and after training was significantly different $(p<0.05)$. The neuron shown in $\mathbf{a}$ is the same neuron shown in Fig. 3a, b. In $\mathbf{c}$ and $\mathbf{h}$, discharge modulation of the same neurons is connected by lines. In $\mathbf{d}$ and $\mathbf{i}$, eye velocity while recoding from the same neurons is connected by lines. Asterisks in $\mathbf{c}-\mathbf{e}$ and $\mathbf{h}-\mathbf{i}$ indicate data for the neurons shown in $\mathbf{a}$ and $\mathbf{f}$, respectively. In $\mathbf{c}$ and $\mathbf{h}$, discharge modulation during vestibular stimulation alone without a target was subtracted from the discharge modulation during the last $5 \mathrm{~min}$ of training (after) for each neuron. For further explanation, see text 


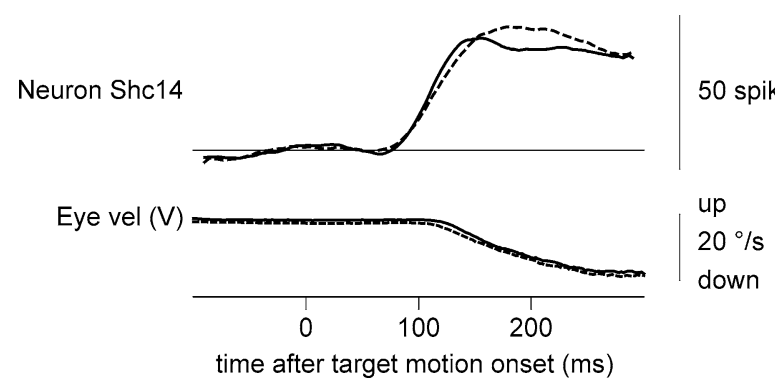

Fig. 6 Comparison of eye velocity and discharge modulation during target only condition before and after training. Time course of the spike density function of a representative neuron and simultaneously recorded eye velocity during vertical pursuit before (dashed) and after training (solid) without vestibular stimulation

vestibular stimulation. Figure 6 illustrates discharge of a representative neuron together with simultaneously recorded vertical eye velocity. Compared to the control discharge modulation and vertical eye velocity before training (Fig. 6, dashed lines), no significant change was observed during last $5 \mathrm{~min}$ of training (Fig. 6, solid lines). A total of eight neurons were tested. None of them showed a significant change in discharge modulation and simultaneously recorded vertical eye velocity after training.

\section{Recording location}

Figure 7 illustrates the recording tracks of monkey Si. Responding neurons were found mostly in the fundus of the arcuate sulcus. These areas were similar to those reported in previous studies for pursuit neurons responsive to whole body rotation (Fukushima et al. 2000a; Ebata et al. 2004). Another monkey ( $\mathrm{Sh}$ ) is still being used for other experiments, but the discharge characteristics of pursuit neurons of this monkey (e.g., Fig. 3) were similar to those of

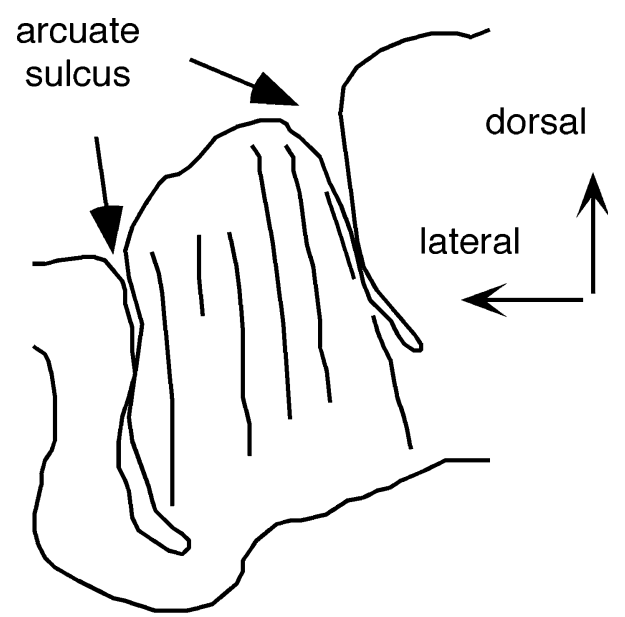

Fig. 7 Recording tracks of monkey Si. Responding neurons were found near the bottom of each track in the fundus of the arcuate sulcus monkey Si and also to those of pursuit neurons in previous studies in which recording locations were confirmed histologically to be within the caudal FEF (e.g., MacAvoy et al. 1991; Gottlieb et al. 1994; Tanaka and Fukushima 1998; Fukushima et al. 2000a, 2002; Akao et al. 2009). The similarity in the recording locations in stereotaxic coordinates and discharge characteristics suggest that recording in monkey Sh also was from the caudal FEF.

\section{Discussion}

The present study has shown that the discharge of $61 \%$ of pursuit neurons tested (14/23) in the caudal FEF exhibited a significant change in discharge modulation during crossaxis vestibular-pursuit training; the latencies to the onset of vertical target motion shortened (Fig. 4a) and the magnitude of initial discharge modulation increased (Fig. 5a, c). These changes occurred in association with the change in simultaneously recorded vertical eye velocity (Figs. 2c, 4ab, 5a-d). However, contrary to our expectation, only a fraction of the neurons tested $(4 / 23=17 \%)$ discharged early enough to initiate such adaptive pursuit (Fig. 4c). Moreover, when the discharge was aligned on the onset of vertical eye velocity, the majority of them (19/23) exhibited a decrease in discharge modulation during the initial $100 \mathrm{~ms}$ after the onset of eye velocity (Fig. 5e).

In the present study, a significant change was minimally induced in the eye velocity and FEF pursuit neuron activity after training by vestibular stimulation alone in complete darkness (Figs. 1e, 3d). This is in contrast to previous studies in which a significant eye velocity change was observed after training by vestibular stimulation alone applied in the plane orthogonal to target motion (Fukushima et al. 1996b, 2000b, 2001a, b; Sato et al. 1999; Tsubuku et al. 2006; cf. Walker and Zee 2002; Fukushima et al. 2006 for a review). We think that this difference was due to the differences in the previous and present training as follows. First, the task conditions were different. In the present (but not previous) training, the initial slow horizontal target motion component was added [Fig. 1a, target pos $(\mathrm{H})$ ]. As a result, identical chair rotation (e.g., toward right) was combined with rightward/upward or leftward/upward target motion (Fig. 1a). This horizontal target motion direction that was combined randomly with chair rotation direction may have interfered with predictive vertical eye movements induced by chair rotation. We used this task condition in the present study to exclude any possible interference from fixationrelated discharge modulation (Goldreich et al. 1992; Schwartz and Lisberger 1994; Fukushima et al. 1996a; Izawa et al. 2004). Notice that chair rotation applied in the plane orthogonal to the pursuit preferred directions induce minimal discharge modulation of FEF pursuit neurons in normal 
monkeys, because pursuit preferred directions are similar to vestibular preferred directions (Fukushima et al. 2000a; Akao et al. 2007).

Second, training duration in previous and present training was different. Because the purpose of the present study was to examine discharge of FEF pursuit neurons during cross-axis vestibular-pursuit training, we restricted the recording period to $45 \mathrm{~min}$ in order to compare discharge of the same neurons before and after training, although some neurons were lost after 25 min of training. In contrast, previous studies recorded only eye movements, and training was continued typically over $1-2 \mathrm{~h}$ in each session (e.g., Fukushima et al. 1996b, 2001a).

Despite these differences in training, there were similarities in eye movement responses in the present and previous studies. First, in both studies the monkeys exhibited shorter latencies and larger initial pursuit eye velocities after training in response to vertical target motion synchronized with vestibular stimulation (e.g., Figs. 2a, 4b, 5d; Fukushima et al. 2001a; Tsubuku et al. 2006). Second, significant change was not observed in eye velocity during pursuit to target motion alone without vestibular stimulation after cross-axis training (Fig. 6; also Fukushima et al. 2001a; Tsubuku et al. 2006). These results suggest that neural mechanisms for predictive pursuit during vestibular stimulation in the presence of a pursuit target in previous and present studies were similar.

In the present study, significant change was not observed in the activity of FEF pursuit neurons during pursuit alone after training, consistent with the lack of the significant change in pursuit eye velocity (Fig. 6). The present results that vestibular stimulation alone after training induced minimal change in FEF pursuit neuron activity (e.g., Fig. 3d) suggest that vestibular inputs alone were insufficient to induce predictive changes. Thus, our results taken together suggest that adaptive change for pursuit prediction in our task was induced by vestibular inputs in the presence of a pursuit target at the sites where vestibular and pursuit inputs converged (see "Introduction"). Although in the present study latencies of $61 \%$ of FEF pursuit neurons tested $(14 / 23)$ to the onset of target motion shortened during vestibular-pursuit training in association with latency shortening of vertical eye velocity (Figs. 3a-b, 4a-d), only $17 \%$ of the neurons (4/23) discharged before the onset of simultaneously recorded eye velocity responses when discharge was aligned on the onset of vertical eye velocity (Fig. 4c). Moreover, the majority of them (19/23) exhibited a decrease in discharge modulation during the initial $100 \mathrm{~ms}$ after the onset of eye velocity responses (Fig. 5e). These results suggest that FEF pursuit neurons are unlikely to be involved in the initial stage of generating predictive pursuit eye movements induced by vestibular-pursuit train- ing. Brain areas other than the FEF must be involved in this early stage of predictive pursuit generation.

As for the four neurons that discharged before the onset of simultaneously recorded vertical eye velocity, we cannot determine in the present study whether the change occurred first in the caudal FEF or if the FEF received the change that had occurred in upstream pathways. A potential area in the cerebral cortex would be the SEF (see "Introduction"; for a review, see Fukushima et al. 2006). Single neuron recording from the SEF is necessary to check this possibility. Although medial superior temporal area (MST) and ventral intraparietal area (VIP) are also known to contain pursuit signals and vestibular signals (MST: Sakata et al. 1983; Kawano et al. 1984; Thier and Erickson 1992; Akao et al. 2005b; VIP: Colby et al. 1993; Bremmer et al. 2002), pursuit-related neurons in these areas typically discharge after the onset of smooth pursuit eye movements (e.g., Komatsu and Wurtz 1988; Schlack et al. 2003), suggesting that it is unlikely that neurons in these areas could provide a command signal for predictive pursuit eye movements.

Predictive smooth pursuit in humans is reported to be largely preserved even after large cortical lesions including the frontal cortex (Lekwuwa and Barnes 1996). This result suggests that predictive pursuit functions must be distributed in multiple circuits including those frontal cortical structures and feedback circuits possibly from the cerebellum (see Fukushima 2003 for a review). To explain known smooth pursuit characteristics, Robinson (1982) proposed a model in which the smooth pursuit system uses an internal representation of target velocity calculated from retinal image-slip-velocity of a target and efference copy of eye velocity (also Robinson et al. 1986). The importance of this positive feedback loop in maintenance of smooth pursuit has been supported in many other models that followed (see Leigh and Zee 2006 for a review). Although the neural substrate of such feedback loop is still unknown, it may include feedback from the cerebellum. The present results showing that the discharge of $61 \%$ of FEF pursuit neurons tested exhibited a significant increase in discharge modulation during cross-axis vestibular-pursuit training in association with the increase in simultaneously recorded vertical eye velocity (Figs. 2, 4a-b, 5a-d) suggest that these neurons participated in some aspect of predictive pursuit. An increase in discharge modulation after training that occurred after $100 \mathrm{~ms}$ following the onset of pursuit eye velocity (Fig. 5c) may suggest that these neurons still could participate in the maintenance of predictive pursuit. This increase in discharge modulation may be induced by internal feedback (cf. Tanaka and Lisberger 2002). The periodicity in firing that appeared during training (Fig. 2b, 20-25 min) may have reflected discharge through such feedback loop. 
Acknowledgments We thank Dr. Chris R. S. Kaneko and the anonymous reviewers for their valuable comments on the manuscript. This research was supported by Grant-in-Aid for Scientific Research on Priority Areas (System study on higher-order brain functions) (17022001) and (C) (20500351) from the MEXT of Japan. Mr. Hiroshi Saito participated in early experiments.

Open Access This article is distributed under the terms of the Creative Commons Attribution Noncommercial License which permits any noncommercial use, distribution, and reproduction in any medium, provided the original author(s) and source are credited.

\section{References}

Akao T, Kurkin S, Fukushima J, Fukushima K (2005a) Visual and vergence eye movement related responses of pursuit neurons in the caudal frontal eye fields to motion-in-depth stimuli. Exp Brain Res 164:92-108

Akao T, Mustari MJ, Fukushima J, Kurkin S, Fukushima K (2005b) Discharge characteristics of pursuit neurons in MST during vergence eye movements. J Neurophysiol 93:2415-2434

Akao T, Saito H, Fukushima J, Kurkin S, Fukushima K (2007) Latency of vestibular responses of pursuit neurons in the caudal frontal eye fields to whole body rotation. Exp Brain Res 177: 400-410

Akao T, Kurkin S, Fukushima J, Fukushima K (2009) Otolith inputs to pursuit neurons in the frontal eye fields of alert monkeys. Exp Brain Res 193:455-466

Barnes GR, Asselman PT (1991) The mechanism of prediction in human smooth pursuit eye movements. J Physiol 439:439-461

Bremmer F, Klam F, Duhamel JR, Ben Hamed S, Graf W (2002) Visual-vestibular interactive responses in the macaque ventral intraparietal area (VIP). Eur J Neurosci 16:1569-1586

Colby CL, Duhamel L, Goldberg ME (1993) Ventral intraparietal area of macaque: anatomic location and visual response properties. J Neurophysiol 69:902-914

Ebata S, Sugiuchi Y, Izawa Y, Shinomiya K, Shinoda Y (2004) Vestibular projection to the periarcuate cortex in the monkey. Neurosci Res 49:55-68

Fuchs AF, Robinson DA (1966) A method for measuring horizontal and vertical eye movements chronically in the monkey. J Appl Physiol 21:1068-1070

Fujiwara K, Takeichi N, Tsubuku T, Fukuda S, Fukushima K (2007) Adaptive changes in smooth pursuit eye movement induced by vestibular inputs and activity of pursuit neurons in the frontal eye fields (in Japanese). Equilibrium Res 66:241 (abstr)

Fukushima K (2003) Frontal cortical control of smooth-pursuit. Curr Opin Neurobiol 13:647-654

Fukushima K, Tanaka M, Suzuki Y, Fukushima J, Yoshida T (1996a) Adaptive changes in human smooth pursuit eye movement. Neurosci Res 25:391-398

Fukushima K, Fukushima J, Chin S, Tsunekawa H, Kaneko CRS (1996b) Cross-axis vestibulo-ocular reflex induced by pursuit training in alert monkeys. Neurosci Res 25:255-265

Fukushima K, Sato T, Fukushima J, Shinmei Y, Kaneko CR (2000a) Activity of smooth pursuit-related neurons in the monkey periarcuate cortex during pursuit and passive whole-body rotation. J Neurophysiol 83:563-587

Fukushima K, Sato T, Fukushima J, Kurkin S (2000b) Cross-axis VOR induced by pursuit training in monkeys: further properties of adaptive responses. Arch Ital Biol 138:49-61

Fukushima K, Wells SG, Takeichi N, Yamanobe T, Fukushima J (2001a) Adaptive changes in smooth pursuit eye movement induced by pursuit-vestibular interaction training in monkeys. Exp Brain Res 139:473-481
Fukushima K, Fukushima J, Yamanobe T, Shinmei Y, Kurkin S (2001b) Adaptive eye movements induced by cross-axis pursuitvestibular interactions in trained monkeys. Acta Otolaryngol Suppl 545:73-79

Fukushima K, Yamanobe T, Shinmei Y, Fukushima J (2002) Predictive responses of peri-arcuate pursuit neurons to visual target motion. Exp Brain Res 145:104-120

Fukushima J, Akao T, Takeichi N, Kurkin S, Kaneko CR, Fukushima K (2004) Pursuit-related neurons in the supplementary eye fields: discharge during pursuit and passive whole body rotation. J Neurophysiol 91:2809-2825

Fukushima J, Akao T, Kurkin S, Kaneko CRS, Fukushima K (2006) The vestibular-related frontal cortex and its role in smooth-pursuit eye movements and vestibular-pursuit interactions. J Vestibular Res 16:1-22

Fukushima K, Akao T, Shichinohe N, Nitta T, Kurkin S, Fukushima J (2008) Predictive signals in the pursuit area of the monkey frontal eye fields. Prog Brain Res 171:433-440

Goldreich D, Krauzlis RJ, Lisberger SG (1992) Effect of changing feedback delay on spontaneous oscillations in smooth pursuit eye movements of monkeys. J Neurophysiol 67:625-638

Gottlieb JP, MacAvoy MG, Bruce CJ (1994) Neural responses related to smooth pursuit eye movements and their correspondence with electrically elicited slow eye movements in the primate frontal eye field. J Neurophysiol 72:1634-1653

Heinen SJ, Liu M (1997) Single-neuron activity in the dorsomedial frontal cortex during smooth-pursuit eye movements to predictable target motion. Visual Neurosci 14:853-865

Izawa Y, Suzuki H, Shinoda Y (2004) Suppression of visually and memory-guided saccades induced by electrical stimulation of the monkey frontal eye field. II. Suppression of bilateral saccades. J Neurophysiol 92:2261-2273

Kawano K, Sasaki M, Yamashita M (1984) Response properties of neurons in posterior parietal cortex of monkey during visualvestibular stimulation. I. Visual tracking neurons. J Neurophysiol $51: 340-351$

Komatsu H, Wurtz RH (1988) Relation of cortical areas MT and MST to pursuit eye movements. I. Localization and visual properties of neurons. J Neurophysiol 60:580-603

Kurkin S, Akao T, Fukushima J, Fukushima K (2009) Discharge of pursuit-related neurons in the caudal part of the frontal eye fields in juvenile monkeys with up-down pursuit asymmetry. Exp Brain Res 193:181-188

Leigh RJ, Zee DS (2006) The neurology of eye movements, 4th edn. Oxford University Press, New York

Lekwuwa GU, Barnes GR (1996) Cerebral control of eye movements. II. Timing of anticipatory eye movements, predictive pursuit and phase errors in focal cerebral lesions. Brain 119:491-505

MacAvoy MG, Gottlieb JP, Bruce CJ (1991) Smooth pursuit eye movement representation in the primate frontal eye field. Cereb Cortex 1:95-102

Richmond BJ, Optican LM (1987) Temporal encoding of two-dimensional patterns by single units in primate inferior temporal cortex. II. Quantification of response waveform. J Neurophysiol 57:147-161

Richmond BJ, Optican LM, Podell M, Spitzer H (1987) Temporal encoding of two-dimensional patterns by single units in primate inferior temporal cortex. I. Response characteristics. J Neurophysiol 57:132-146

Robinson DA (1982) A model of cancellation of the vestibulo-ocular reflex. In: Lennerstrand G, Zee DS, Keller EL (eds) Functional basis of ocular motility disorders. Pergamon press, Oxford, pp 5-13

Robinson DA, Gordon JL, Gordon SE (1986) A model of the smooth pursuit eye movement system. Biol Cybern 55:43-57

Sakata H, Shibutani H, Kawano K (1983) Functional properties of visual tracking neurons in posterior parietal association cortex of the monkey. J Neurophysiol 49:1364-1380 
Sato T, Yokoyama R, Fukushima J, Fukushima K (1999) Latency of cross-axis vestibulo-ocular reflex induced by pursuit training in monkeys. Neurosci Res 33:65-70

Schlack A, Hoffmann K-P, Bremmer F (2003) Selectivity of macaque ventral intraparietal area (area VIP) for smooth pursuit eye movements. J Physiol 551:551-561

Schwartz JD, Lisberger SG (1994) Initial tracking conditions modulate the gain of visual-motor transmission for smooth pursuit eye movements in monkeys. Visual Neurosci 11:411-424

Singh A, Thau GE, Raphan T, Cohen B (1981) Detection of saccades by a maximum likelihood ratio criterion. In: Proceedings of the 34th Annual Conference of Engineering Biology, Houston, $\mathrm{p} 136$

Szucs A (1998) Applications of the spike density function in analysis of neuronal firing patterns. J Neurosci Methods 81:159-167
Tanaka M, Fukushima K (1998) Neuronal responses related to smooth pursuit eye movements in the periarcuate cortical area of monkeys. J Neurophysiol 80:28-47

Tanaka M, Lisberger SG (2002) Role of arcuate frontal cortex of monkeys in smooth pursuit eye movements. II. Relation to vector averaging pursuit. J Neurophysiol 87:2700-2714

Thier P, Erickson RG (1992) Responses of visual-tracking neurons from cortical area MST-1 to visual, eye and head motion. Eur J Neurosci 4:539-553

Tsubuku T, Akao T, Kurkin SA, Fukushima K (2006) Prediction in the timing of pursuit eye movement initiation revealed by cross-axis vestibular-pursuit training in monkeys. Exp Brain Res 168:427-435

Walker MF, Zee DS (2002) Rectified cross-axis adaptation of the vestibulo-ocular reflex in rhesus monkey. Ann N Y Acad Sci 956:543-545 